\title{
Chronic Ovarian Torsion Treated Laparoscopically
}

\author{
M. Sami Walid, MD, $\mathrm{PhD}^{1}$; Richard L. Heaton, MD, FACOG ${ }^{1}$
}

Keywords: Ovarian Torsion; Laparoscopy

\begin{abstract}
Ovarian torsion is a rare gynecological emergency that may elude diagnosis leading to delayed operative intervention and ovarian resection. We report a 39 year old female who presented with acute one-sided abdominal pain whose diagnosis was missed by the emergency physician resulting in loss of the ovary. The surgery was performed by the gynecologist laparoscopically.

${ }^{1}$ Heart of Georgia Women's Center, 209 Green Street, Warner Robins, GA.
\end{abstract}

\section{Introduction}

Ovarian torsion is a rare gynecological emergency that may elude diagnosis leading to delayed operative intervention and ovarian loss. The patient usually suffers from symptoms for 3 days and the diagnosis is usually at surgery ${ }^{1}$.

\section{Case Report}

A 39 year old female, gravida 3, para 1 , came to the emergency room with right-sided pelvic pain. She had a history of hysterectomy and left salpingo-oophorectomy five years ago. She was prescribed an antiinflammatory drug and discharged home. Three days later she returned with increasing right-sided pelvic pain. Abdominal palpation elicited rebound pain in the right lower quadrant. Ultrasound imaging showed an enlarged right ovary. Diagnostic laparoscopy was performed which revealed a black (infarcted) and torsed right ovary with avulsion of the parietal peritoneum (see picture). The infracted ovary was removed laparoscopically.

Please cite this paper as: Walid MS, Heaton RL. Chronic Ovarian Torsion Treated Laparoscopically. Proc Obstet Gynecol. 2011 Apr;1(3):Article 1[3 p.]. 2010 December [Epub ahead of print]. Available from: http://ir.uiowa.edu/pog/. Free full text article.

Corresponding author: Richard L. Heaton, Heart of Georgia Women's Center, 209 Green Street, Warner Robins, GA. riclheaton@yahoo.com

This is an Open Access article distributed under the terms of the Creative Commons Attribution 3.0 Unported License (http://creativecommons.org/licenses/by/3.0), which permits unrestricted use, distribution, and reproduction in any medium, provided the original work is properly cited. 


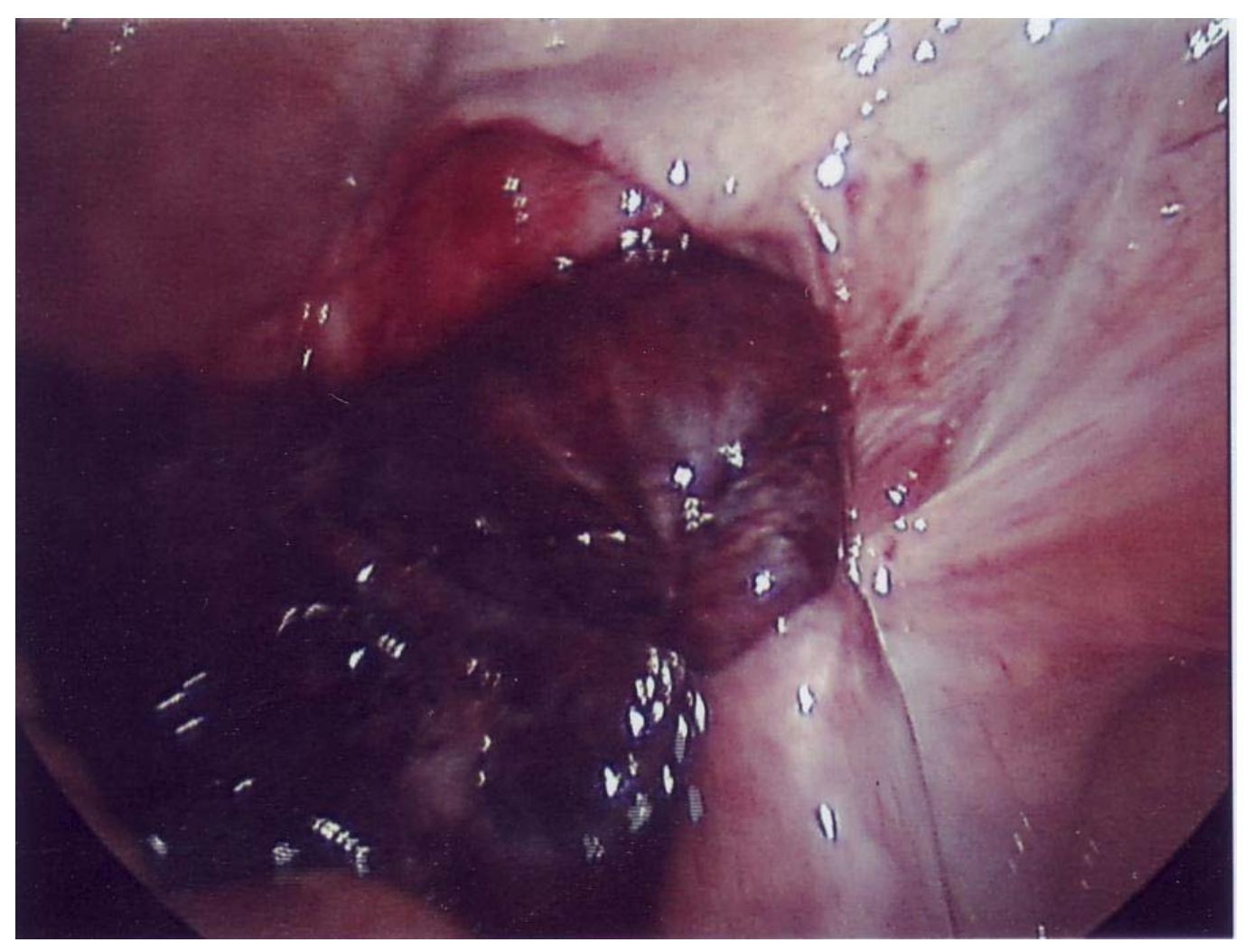

Laparoscopic picture showing the torsed right ovary with avulsion of the parietal peritoneum.

\section{Discussion}

Laparoscopic treatment of ovarian torsion is a relatively simple procedure for an advanced minimal invasive surgeon. Therefore, open laparotomy should be discouraged if access to minimal invasive surgery is available. The most important part of the procedure is to begin with locating the ureter at the pelvic brim then open the peritoneum at this level and dissect the ureter out past the torsion to assure that it in not injured in taking the pedicle to remove the necrotic ovary.

Chronic Ovarian Torsion
Over a period of nearly three decades we have encountered a handful of ovarian torsion cases. Ovarian torsion cases should be differentiated from hemorrhagic ovarian cyst cases. Sonography is usually not reliable in the diagnosis or exclusion of this entity ${ }^{2}$. From our experience, an edematous ovary on ultrasound that appears larger on successive investigations in association with a slightly elevated white blood count ${ }^{2}$ seems to be more suggestive of ovarian torsion. A hemorrhagic ovarian cyst usually reaches its largest diameter quickly and often on the first ultrasound 


\section{Epub ahead of print}

investigation. A hemorrhagic ovarian cyst is also less likely to be associated with an elevated white blood count. Sometimes, a positive whirlpool sign in the twisted vascular pedicle of the ovary can be detected using color Doppler sonography ${ }^{3}$. This is considered "the most definitive sign" of ovarian torsion ${ }^{3,4}$.

\section{Conclusion}

Ovarian torsion has an incidence of $3 \%$ among acute gynecological admissions and is frequently missed at first presentation $^{5}$. It should always be suspected when the patient is female with acute abdomen and one-sided pelvic pain. In our patient's case, an absent uterus and absent left ovary should have alerted the emergency doctor to the possibility of ovarian torsion in the remaining right ovary. A strategy of nonhesitant use of diagnostic laparoscopy in female acute abdomen cases may improve detection rates and ovarian salvage odds $^{2}$. A study into the rate of ovarian torsion occurrence in patients with a history of hysterectomy and the culpability of disturbing the anchoring system of the ovary in the pathomechanism of this acute misfortune is warranted.

\section{References}

1. White $M$, Stella J. Ovarian torsion: 10-year perspective. Emerg Med Australas. 2005 Jun;17(3):231-7.

2. Oltmann SC, Fischer A, Barber R, Huang R, Hicks B, Garcia N. Cannot exclude torsion--a 15-year review. J Pediatr Surg. 2009 Jun;44(6):12126; discussion 1217.

3. Lee EJ, Kwon HC, Joo HJ, Suh JH, Fleischer AC. Diagnosis of ovarian torsion with color Doppler sonography: depiction of twisted vascular pedicle. J Ultrasound Med. 1998 Feb;17(2):83-9.

4. Vijayaraghavan SB. Sonographic whirlpool sign in ovarian torsion. J Ultrasound Med.

2004

Dec;23(12):1643-9; quiz 1650-1.

5. Becker JH, de Graaff J, Vos CM. Torsion of the ovary: a known but frequently missed diagnosis. Eur $\mathrm{J}$ Emerg Med. 2009 Jun;16(3):124-6. 